of Heaviside's "Electro-magnetic Theory". In 1957, in a house at Paignton, once occupied by Heaviside, a further collection of his manuscripts was discovered; these are now described by Mr. Josephs in the Institution's Monograph No. 319.

Some of these notes seem to be corrections and amplifications of Vol. 2 of the "Electro-magnetic Theory", which Heaviside would have incorporated in this volume had not the publishers insisted on a simple reprinting of the articles in The Electrician which formed the substance of the treatise. They show the emphasis placed by Heaviside on a symmetrical formulation of the electro-magnetic equations which, while not improperly called Maxwell's, seem to have been first stated explicitly by Heaviside; and they show his interest in, and attempts to establish, a unified field theory embracing both electromagnetic and gravitational phenomena, based on a general energy-flow equation. They also contain much about Heaviside's operational calculus, particularly in connexion with fractional differentiation, and suggest that Heaviside explored the idea of treating $p$ not simply as the differentiation operator, but as the parameter in an integral transform, a point of view much closer to present-day interpretations of his calculus.

Whether or not a careful analysis of the large mass of papers found at Paignton would now be worth while, Mr. Joseph's article can only increase respect for Heaviside as an unorthodox but brilliant mathematician. Mr. Joseph revives the comment that Heaviside's operational methods made no appeal to mathematicians because such methods did not conform to the standards of rigour fashionable at the time; this is not in agreement with the conclusions reached by Prof. J. L. B. Cooper, who has discussed the point in considerable detail in an article in the Mathematical Gazette for February 1952.

\section{Isaac Newton Telescope}

FinanCIaL approval has been given to proceed with the construction of the proposed 98-in. Isaac Newton telescope which will be erected at the Royal Greenwich Observatory, Herstmonceux. This project was sponsored by the Royal Society, at the instance of the Royal Astronomical Society, and approved in principle by the Treasury and the Board of Admiralty in 1946. The design of the instrument received careful consideration by the scientists concerned, and development work was undertaken. In 1955 it was decided that the new Astronomer Royal should, on appointment, be given the opportunity of considering the final form of the telescope, and a quotation for construction of the instrument was obtained in November 1956. Owing to lack of funds, it was not then possible, however, to proceed with the actual construction. The new telescope, which will be the largest in the United Kingdom, will be used by visiting astronomers as well as by the staff at the Royal Observatory. The cost of the whole project, including erection and preparation of the site, is estimated at $£ 660,000$ at present prices, and will take five to six years to complete. Expenditure will be shared equally between the Treasury and the Admiralty.

The Isaac Newton telescope will be designed for use at the prime focus, Cassegrain focus, and coudé focus. Special attempts will be made to design a Cassegrain combination suitable for astrometry. The telescope tube will be held in an open fork with no north support ; the Cassegrain focus will be reached from a platform carried by the telescope itself. It is intended to carry out an extensive programme of parallax work at the Cassegrain focus, using the large aperture to observe much fainter stars than are accessible to the comparatively small refractors now used on parallax work. The telescope is very flexible as regards choice of programme, and at present it is intended to concentrate the spectroscopic programmes on the precise determination of radial velocity, especially in those cases where individual stellar velocities may be supposed to be important in connexion with problems in stellar dynamics. In particular, it is planned to provide a large grating spectrograph at the coudé focus, with cameras of approximately 100-in., 60-in. and 30-in. focus. The prime focus will be available for the direct photography of stellar fields of interest in radio astronomy, and will be equipped with a fieldcorrecting lens.

\section{Effects of the Aurora on Radio Reception}

A RESEARCH station to study the effects of the aurora on radio reception is to be set up on the site of a former radar station at Hillhead, near Fraserburgh, in Aberdeenshire. This investigation, part of a programme of ionospheric studies, will be undertaken by the Stanford Research Institute of America in collaboration with scientists from the Royal Radar Establishment of the Ministry of Supply. Observations will be made with a large parabolic reflector about $140 \mathrm{ft}$. in diameter brought from America. The experiments are expected to start in the late spring or early summer and will last one or two years. The cost will be borne by the Stanford Research Institute. The Aberdeenshire site has been chosen because of its geographical suitability for the study of auroral phenomena. There will be no radiation danger, and interference with radio and television will be prevented by the operating conditions laid down for the installation. There should be no restrictions on agricultural work, except that the vicinity of the installation must be protected from damage by livestock.

\section{Biochemistry at the Royal College of Surgeons}

IN days gone by, surgical shock was interpreted in terms of vaso-motor reactions and nervous responses to the stimulus of a severe injury. To-day attention is directed rather to the metabolic (chemical) response to injury, and since a chemical mechanism is now known to underlie the phenomena of shock, its treatment must be chemical also. Hitherto the process of wound healing has been regarded as a matter of cellular and vascular processes, explicable on a histological basis. But there was a mysterious initial phase when nothing could be seen to be going on in the wound, sometimes referred to as 'the lag period'. Recent research has shown that during these early days there is intense chemical activity in the wound, resulting in the accumulation of certain substances which are essential for the natural process of repair and without which the lag period may be indefinitely prolonged. There are profound biochemical changes in patients suffering from cancer, and furthermore there are tumours which are known to depend for their growth upon certain chemical substances, the deprivation of which can result in such diminution of growth as to suggest the possibility of treating cancer by chemical means. These are only examples to indicate the importance of biochemistry in surgery to-day, and it is no wonder 